\title{
A small UAV deviation control roll research
}

\author{
Tianxiang $\mathrm{Hu}$ \\ College of Mechatronic Engineering, Nanjing Institute of Industry Technology, Nanjing 210023, \\ China \\ Email: hutuson@163.com
}

Keywords: UAV; Roll; Flying control; Rectify deviation

Abstract: This paper according to the small UAV structure characteristics, the taxiing control structure and control law design are studied and proposed front wheel and rudder deviation control scheme and control structure, analysis, including the front landing gear, pavement condition, brake system, wind and other factors for correcting deviation control effect influence, based on take-off and landing and taxiing deviation control take-off characteristics, design the taxiing test scheme.

\section{Introduction}

Wheeled landing approach is large and medium-sized UAV using an important landing approach, the cost is expensive, landing gear system is more complex, and is equipped with brake system, so ground of ground taxiing control the main wheel differential brake and control method of rudder combined correction. However, for small wheeled landing mode of the UAV, due to its light weight, low cost, easy to use and maintenance, the landing gear design is generally relatively simple. Due to the front wheel and the differential brake can implement the deviation control, front wheel steering control with quick response, no additional resistance, roll a short distance, differential brake on the landing gear brake system requirements higher, thus greatly increased the landing gear of the weight and cost. Using the front wheel steering control of the UAV, can not install the brake system or modified simple brake system for reduction, because the structure is simple, so the small wheeled landing UAV usually use this way of deviation correction.

In the present study on man-machine taxiing characteristics, mostly taxiing mathematical model based on the accurate of taxiing model of UAV Based on. However, because of the UAV in taxiing process of influencing factors, such as front landing gear overhead back, pavement condition, wind or other. Therefore, it is very difficult to establish accurate mathematical model. Especially small unmanned aerial vehicle (UAV), to carry out wind tunnel experiment is not only long life cycle, and costly, through the taxiing test parameter adjustment is small UAV control law design is an important method.

\section{Ground control scheme and structure}

UAV lateral motion information into the front wheel steering deviation control law and rudder deviation control law, front wheel steering control and rudder control. At the same time to UAV, by the two control channel control law operation produced front wheel steering deviation control instruction and rudder rectifying deviation control instruction, in low-speed taxiing section, front wheel correction efficiency is high, the rudder authority rate lower, front wheel correction plays a 
major role; in the high-speed range, the rudder authority higher rate, because of a surge in the UAV lift, the pressure of front wheels on the road is very small, so a front wheel steering correction efficiency is very low, the rudder play main role. In order to ensure seamless handover of UAV in taxiing of front wheel deviation control and rudder deviation control rudder deviation control law through the distributor and steering control law together to produce steering control commands, enter into the front wheel steering mechanism of deflection torque control lateral movement of the UAV. The UAV lateralization in a safe range. Taxiing in rectifying process, if appear out of the runway and other emergency situations, the brake control law based on the instruction of lateral distance and sliding speed are brake, braking instruction enters into the brake mechanism to control the UAV taxiing speed, ensure that the UAV sliding safety. Here, the brake does not participate in the lateral control.

The lateral offset based feedback signal, because the front wheel steering control response is more sensitive, the lateral velocity and yaw rate control to improve the dynamic performance, these signals into the front wheel steering correction control law, the front wheel steering correction control law generates front wheel steering control command; in addition, at the same time as the main lateral offset feedback signal and the yaw angle, yaw rate in rudder deviation control law direction, because the relatively slow response of rudder control, where the yaw angle and yaw rate to improve the dynamic performance of control, the rudder deflection correcting control law generates instructions to enter the rudder rudder loop make rudder yaw moment generated deviation. In order to realize the smooth transition between front wheel steering control and directional control, the design of the distributor is that the control parameters of the control parameters of the control parameters of the steering control system are generated according to a certain transmission ratio. Taxiing in rectifying process and roll within the loop at the same time, to prevent the aircraft in the rectification movement due to turn centrifugal force, wind or other factors lead to the roll angle is too large, so that the risk of rollover occurred in the process of turning.

Taxiing stage, UAV lateral control law for specific expression:

$$
\begin{gathered}
\delta_{\alpha}=K_{\alpha}^{P} P+K_{\alpha}^{\phi}\left(\phi-\phi_{c}\right) \\
\delta_{f}=K_{f}^{R} R+K_{f}^{Y^{\&}} \vartheta+K_{f}^{Y} \\
\delta_{r}=K_{r}^{R} R+K_{r}^{\Psi}\left(\Psi-\Psi_{c}\right)+K_{r}^{Y} Y
\end{gathered}
$$

$\delta_{\mathrm{a}}$ is for aileron rudder offset value, $\mathrm{P}$ is the rolling angular rate, $\phi$ for roll angle, $\phi_{\mathrm{c}}$ is the roll angle commands, $\mathrm{K}_{a}^{p}$ and $\mathrm{K}_{a}^{\phi}$ are aileron differential and the proportional control gain.

$\delta_{\mathrm{f}}$ is front wheel steering rudder deflection angle, $R$ is the yaw rate, $Y$ is relative to the runway center line of the lateral offset, $\mathrm{Y}^{\&}$ is the lateralization rate available formula obtained:

$$
Y^{\&}=V_{g} \times \sin \left(\psi-\psi_{c}\right)
$$

$\mathrm{V}_{\mathrm{g}}$ is the ground speed, $\psi_{\mathrm{c}}$ is UAV heading command, $\mathrm{C}$ is the current direction of UAV 
Ground speed.

$K_{\mathrm{f}}^{R}$ is the front wheel, the yaw angle rate control gain, $\mathrm{K}_{\mathrm{f}}^{Y^{\&}}$ is the front wheel side velocity control gain, and $\mathrm{K}_{\mathrm{f}}^{Y}$ is the front wheel side offset control gain.

$\delta_{\mathrm{r}}$ is rudder angle, $\quad \mathrm{K}_{r}^{R}$ is the rudder yaw angle rate control gain, and $\mathrm{K}_{\mathrm{r}}^{\psi}$ is the rudder yaw angle control gain, $\mathrm{K}_{r}^{Y}$ is the rudder of a lateral control gain.

$\delta^{\mathrm{a}} 、 \delta^{f}$ and $\delta^{r}$ are obtained after the limit, $\delta^{a c} 、 \delta^{f c} 、 \delta^{\text {rc }}$ to the output circuit

The design details are as follows:

$$
\begin{aligned}
& K_{r}^{R}=K_{r}^{R} \times K_{f}^{r} \\
& K_{f}^{Y^{\&}}=K_{r}^{\psi} \times K_{f}^{r} \\
& K_{f}^{Y}=K_{r}^{Y} \times K_{f}^{r}
\end{aligned}
$$

$\mathrm{K}_{\mathrm{f}}^{Y}$ is the distribution coefficient from the rudder to the front wheel.

\section{Ground to adjust the parameters of experimental design}

\section{Lateral deviation control require ments}

Through the taxiing test to debug control parameters, we need to make clear rectifying control requirements. In the control of UAV Ground run the requirements as follows:

(1) the yaw rate can not be too large, that is in the process of running the body does not appear obvious swing;

(2) UAV relative lateral offset to the runway center line to keep in allowable range, prevent taxiing due to runway Road, crosswind disturbance and other factors make no man-machine runway.

(3) during taxiing without man-machine nose heading and the runway centerline heading angle should be in reasonable range, and there should not be a large angle correction or even turning a dangerous situation.

\section{influence factors of deviation correction}

UAV, ground taxiing in, affect the correction control effect of many for the proposed control scheme, in the test process must focus on four aspects: (1)front air back、(2)pavement condition、 (3)brake effect、(4)the gust effect

The direct influence of the size of the front wheel back to the control accuracy, the smaller the front wheel back to the smaller the better control effect. The empty back and the back of the front wheel steering gear and the back of the transmission mechanism, the gap between the transmission rod and the fixed gap between the wheel and the wheel, the two kinds of air return should be 
considered in the landing gear design, the general should be less than $0.5^{\circ}$.

Pavement condition is mainly considered the influence of pavement slope and pavement irregularities. Road gradient and the main landing gear rack two machine tire pressure inconsistent on the control effect of the same, mainly affecting the skating when cornering static error and different runway pavement different slope, even with a runway in UAV back and forth taxiing correction effect is also different. Therefore, no man in taxiing must pass free glide tests without control, continuous commissioning front of zero, to ensure that front zero within the appropriate range and to eliminate side partial static error. The road bump mainly affects the safety running, may lead to a significantly uneven pavement before landing gear damage or UAV rollover.

Brake system, though not for left and right wheel differential correction, but because of the left and right brake mechanism of asymmetry, when in taxiing of emergency deceleration, resulting in UAV nose to brake torque on the larger side thrash, under the situation of fast may make UAV rollover or overshot the runway.

Although the taxiing process time is short, during the period of may by gust effect in man-machine sloshing, taxi trajectories deviate from the runway center line. Lateral deviation control should be timely and smoothly will not control back to the safety range, and would not have a big overshoot.

\section{Test design}

As a result of the UAV in take-off and landing phases into the initial state of the ground taxiing, control parameters test tuning into the focus is different.

The initial conditions for the test of the takeoff section are as follows:

(1) the UAV parked on the runway center line, the lateral offset is almost zero;

(2) the head of the UAV is aligned with the center line of the runway, and the heading deviation of the center line of the nose and the runway is almost zero.

For take-off period of adjusting parameter test, the elevator shall produce nose down moment, prevent because skating speed is too fast, no man-machine accidentally took off from the ground. Send "roll" after the instruction, the UAV taxiing began to accelerate from rest, parking at set speed after. Gradually increasing the speed of setting the sliding speed until the ground velocity can be completed.

UAV ground before subjected to Cross wind Effects, there may be a larger lateral partial and the heading error, so in the design of landing ground taxiing test should be in the UAV to pick up speed skating to produce certain side partial validation of UAV in the rapid sliding state of cornering ability.

\section{Parameter design of correction control law}

Lateral deviation control law design is to determine the parameters control the gain value in the law.

In the low-speed taxiing segment, front wheel correction plays a major role, need to determine $K_{\mathrm{f}}^{R} 、 \mathrm{~K}_{\mathrm{f}}^{Y \&}$ and $\mathrm{K}_{\mathrm{f}}^{Y}$. The three parameters are determined by the process: 
(1) A reference value for the $K_{\mathrm{f}}^{R} 、 \mathrm{~K}_{\mathrm{f}}^{Y \&}$ and $\mathrm{K}_{\mathrm{f}}^{Y}$

(2) $K_{\mathrm{f}}^{R}$ determination: UAV in the take-off process, may head swing, adjust the value of $\mathrm{K}_{\mathrm{f}}^{Y \&}$, head swing within a reasonable range;

(3) $\mathrm{K}_{\mathrm{f}}^{Y}$ determination: UAV in low speed, smooth take-off, but with increasing taxiing speed, body in on both sides of the runway centerline sloshing, namely "the touch" phenomenon, adjust the value of $\mathrm{K}_{\mathrm{f}}^{Y \text { \& }}$, the $\mathrm{UAV}$ a straight taxiing along the runway center line;

$\mathrm{K}_{\mathrm{f}}^{Y}$ is in the roll before, will determine the UAV runway centerline distance, observed during cornering roll control. If the parameter $\mathrm{K}_{\mathrm{f}}^{Y}$ is too large, although the response rate is fast, but it is easy to generate a large overshoot; if the parameters of $\mathrm{K}_{\mathrm{f}}^{Y}$ is too small, the deviation of the response process is slow, it may affect the take-off safety. Therefore, adjust the $\mathrm{K}_{\mathrm{f}}^{Y}$ value, so that the UAV in the case of the initial side or course deviation can be quickly and without overshoot into the runway center line.

In the medium and high speed running, the rudder began to need to determine the value of $\mathrm{K}_{r}^{R} 、 \mathrm{~K}_{\mathrm{r}}^{\psi} 、 \mathrm{~K}_{r}^{Y}$ and $\mathrm{K}_{f}^{r}$ rectification. The design of high speed $\mathrm{K}_{r}^{R}$ and $\mathrm{K}_{\mathrm{r}}^{\psi}$ design section and the speed of $K_{\mathrm{f}}^{R} 、 \mathrm{~K}_{\mathrm{f}}^{Y \&}$ and $\mathrm{K}_{\mathrm{f}}^{Y}$ is similar, and will not go. $\mathrm{K}_{f}^{r}$ and front wheel steering actuator to front wheel drive mechanism, in the same state of the front wheel steering mechanism of conditions, is the middle speed section taxiing to adjust the parameters of the key. If the speed control parameters have been determined, the speed skating there head swing, "the touch" control effect is not too ideal can enlarge or reduce the speed of the corresponding control parameters value, while at the same time reduce or enlarge the $\mathrm{K}_{f}^{r}$ value to achieve both to ensure low speed correction effect is ideal and also corrected taxiing speed.

\section{Test Analys is}

The design method of control law is applied to the design of the control law of a certain type of UAV. The final control law parameters determine the taxiing test as shown in table 1.

Table 1 Parameters of lateral deviation control

\begin{tabular}{c|c|c|c|c|c}
\hline $\begin{array}{c}\text { Paramet } \\
\text { er }\end{array}$ & $K_{f}^{R}$ & $K_{f}^{Y^{\varepsilon}}$ & $K_{f}^{Y}$ & $K_{f}^{r}$ & $\delta_{f c}$ \\
\hline $\begin{array}{c}\text { Numeric } \\
\text { al }\end{array}$ & 0.32 & 1.6 & 1 & 0.375 & $5^{\circ}$ \\
\hline
\end{tabular}

In the first test, no man-machine taxiing before initial laterality for $0.2647 \mathrm{~m}$, initial heading 
error for 211.5 degrees, that is, without man-machine aligned with the runway centerline. UAV takeoff in the process, the maximum lateral offset is $-0.5 \mathrm{~m}$, maximum yaw angle rate for -2.893 degrees per second, the maximum deviation in heading for degree -1.215 , maximum rolling angle degree -0.8951 , UAV in GS to $28.96 \mathrm{~m} / \mathrm{s}$ from take-off, taxiing of low speed and high speed smooth transition, meet the take-off requirements.

In the second experiment, landing taxiing data can be seen, the UAV ground before initial velocity for $21.14 \mathrm{~m} / \mathrm{s}$ and initial lateral partial to $-0.97 \mathrm{~m}$, initial heading error is 0.23 DEG taxiing of maximum lateral offset is $-1.677 \mathrm{~m}$, maximum yaw angle rate for $3.813 \mathrm{deg} / \mathrm{s}$, the maximum deviation in heading for 179 degrees, the maximum rolling angle degree -0.941 , UAV ground quickly and smoothly without overshoot of taxied to the runway center line, stop from the runway centerline of the lateral offset $-0.2648 \mathrm{~m}$, and taxiing of no obvious head swing, ground taxiing of safety, not use emergency brake.

Deviation control law parameters, the realization of the front wheel steering control and rudder control seamless handoff, is designed through the taxiing test parameter adjustment. For UAV actual landing and taxiing test analysis, verify ground taxiing control scheme and the structure is reasonable and feasible, and the control law design is simple, effective and good control effect and meet the small UAV wheeled landing and taxiing requirements.

\section{Conclusions}

For the wheeled landing approach of UAV, this paper puts forward the front wheel and rudder combined correction control scheme and control structure, and is especially suitable for small UAV Ground taxiing; combining rectification control requirements, the analysis of the impact of control scheme rectification effect factors, as a result of these is to carry out the taxiing test and control law design must consider. Also design the takeoff and landing and taxiing test, and discusses the design method of control parameters, avoiding the conventional control design requires accurate modeling of tedious and limitations. The actual flight test shows that the method is feasible, and can effectively improve the design efficiency of the control law of the deviation correction.

\section{Reference}

[1] CAI G W, CHEN B M, PENG K M, et al. Modeling and control system design for a UAV helicopter[C] //Proceedings of the 14th Mediterranean Conference on Control and Automation. Ancona, Italy: IEEE, 2006, 6: 600-606.

[2] JIAO Y S, DU J, WANG X M, et al. H1 state feedback control for UAV Maneuver trajectory tracking[C] //Proceedings of 2010 International Conference on Intelligent Control and Information Processing. Dalian, China: IEEE, 2010, 8: 253-257.

[3] AHMED B, POTA H R, MATT G Flight control of a rotary wing UAV using backstepping[J]. International Journal of Robust and Nonlinear Control, 2010, 20(6): 639 - 658.

[4] LEE C T, TSAI C C. Adaptive backstepping integral control of a small-scale helicopter for airdrop missions [J]. Asian Journal of Control,2010, 12(4): 531-541.

[5] SANCHEZ E N, BECERRA H M, VELEZ C M. Combining fuzzy and PID control for an unmanned helicopter[C] //2005 Annual Meeting of the North American Fuzzy Information 
Processing Society. Detroit,US A: IEEE, 2005, 6: 235-240. 